\title{
Analysis of the Magnus Moment Aerodynamic Characteristics of Rotating Missiles at High Altitudes
}

\author{
Yihang Xu $(\mathbb{D}$, Shaosong Chen $\mathbb{D}$, and Hang Zhou \\ Department of Weapons Science and Technology, Nanjing University of Science and Technology, Nanjing, China \\ Correspondence should be addressed to Shaosong Chen; chenss805@163.com
}

Received 1 December 2020; Revised 22 March 2021; Accepted 30 March 2021; Published 13 April 2021

Academic Editor: Angelo Cervone

Copyright () 2021 Yihang Xu et al. This is an open access article distributed under the Creative Commons Attribution License, which permits unrestricted use, distribution, and reproduction in any medium, provided the original work is properly cited.

\begin{abstract}
The Magnus moment characteristics of rotating missiles with Mach numbers of 1.3 and 1.5 at different altitudes and angles of attack were numerically simulated based on the transition SST model. It was found that the Magnus moment direction of the missiles changed with the increase of the angle of attack. At a low altitude, with the increase of the angle of attack, the Magnus moment direction changed from positive to negative; however, at high altitudes, with the increase of the angle of attack, the Magnus moment direction changed from positive to negative and then again to positive. The Magnus force direction did not change with the change of the altitude and the angle of attack at low angles of attack; however, it changed with altitude at an angle of attack of $30^{\circ}$. When the angle of attack was $20^{\circ}$, the interference of the tail fin to the lateral force of the missile body was different from that for other angles of attack, leading to an increase of the lateral force of the rear part of the missile body. With the increasing altitude, the position of the boundary layer with a larger thickness of the missile body moved forward, making the lateral force distribution of the missile body even. Consequently, Magnus moments generated by different boundary layer thicknesses at the front and rear of the missile body decreased and the Magnus moment generated by the tail fin became larger. As lateral force directions of the missile body and the tail were opposite, the Magnus moment direction changed noticeably. Under a high angle of attack, the Magnus moment direction of the missile body changed with the increasing altitude. The absolute value of the pitch moment coefficient of the missile body decreased with the increasing altitude.
\end{abstract}

\section{Introduction}

Rotating missiles produce the Magnus effect during flight [1]. For nontailed rotor missiles, the asymmetric distortion of the boundary layer and the asymmetric separation of flow in the leeward region are the main causes of the Magnus force [2]. For tailed missiles, the tail fin is coupled with the separation vortex of the missile body and causes a more complex effect $[3,4]$. Although the Magnus force is only $1 / 100$ to $1 / 10$ of the normal force, the Magnus moment has an important effect on the flight stability of a missile. The Magnus moment leads to a coning motion, reduces the missile shooting range, and even causes the flight to fail [5].

Altitude has a great influence on the aerodynamic characteristics of a missile. For a rotating tail-stabilized missile, the Magnus moment is greatly affected by the altitude, leading to a large-angle conical pendulum movement and greatly affecting the flight stability of the missile. The Qinghai-Tibet
Plateau in China has an average elevation of over 4000 meters and experiences low air density and low atmospheric pressure. Hence, many missiles with good flight stability cannot meet stability requirements in this plateau $[6,7]$. The change of aerodynamic characteristics with altitude is the fundamental reason for this phenomenon.

Zhai et al. and Dang et al. $[6,7]$ studied the variation law of aerodynamic characteristics with altitude and its influence on the ballistic characteristics of a tail-stabilized missile. Zhong et al. $[8,9]$ studied the periodic movement of a missile under the conditions of a low density and a high altitude and obtained the range of the Magnus moment coefficient during a stable periodic movement of the missile. Liu et al. [10] analyzed the main reasons for the instability of a certain type of curving tail missile in the Qinghai-Tibet Plateau and put forward an improvement scheme for adopting a straight tail. Zhang et al. [11] propounded that a missile could have a large attack angle cone pendulum movement at a large angle of 
fire. Ma et al. [12] analyzed key differences in the dynamic stability of a rotating missile under plateau and plain conditions and pointed out that the change of the Magnus torque direction was an important factor for the high angle of attack cone pendulum movement of the missile at a high altitude. However, the variation of the Magnus moment characteristics of a rotating missile with altitude is still not clear.

The aerodynamic characteristics of tailed rotating missiles have been also investigated through numerical simulations. Yin et al. [13] carried out numerical calculations on a finner, compared the calculated results with the experimental values of AEDC [14], and found that the calculated values of the transition SST model were close to the experimental values. Therefore, the transition SST model was adopted in the present work.

\section{Numerical Method}

2.1. Numerical Calculation Conditions and Physical Models. The ballistic altitude of a long-range missile ranges between 50 and $80 \mathrm{~km}$. With increasing altitude, variations in pressure, density, and temperature cause a change of the Reynolds number, which has a direct impact on boundary layer transition and flow separation.

Table 1 presents the variation of parameters with altitude. When the altitude is less than $50 \mathrm{~km}$, the continuum hypothesis is true; thus, the Navier-Stokes (N-S) equation can be used. The finner shown in Figure 1 was used for numerical calculations with the following parameters: altitude $=0-60$ $\mathrm{km}, \mathrm{Ma}=1.3$ and $1.5, \alpha=10^{\circ}, 20^{\circ}$, and $30^{\circ}$, relative rotating speed $p=0.02\left(\omega_{x} d / 2 V_{\infty}\right)$, reference length $L_{\text {ref }}=0.04572 \mathrm{~m}$ , reference area $S_{\text {ref }}=0.00164 \mathrm{~m}^{2}$, and center of mass $=$ $0.2286 \mathrm{~m}$ away from the missile head.

2.2. Turbulence Model. In the aerodynamic calculation of a rotating missile, the correct prediction of separation flow and boundary layer transition is the key to accurately calculate the Magnus force. Nobile et al. [15] numerically simulated the aerodynamic characteristics of a rotary wind turbine under strong wind and compared the results of SST $k-\omega, k-\omega$, and $k-\varepsilon$ turbulence models with experimental data. It was found that the SST $k-\omega$ model performed well in an adverse pressure gradient and separation flow. However, the SST $k-\omega$ model causes the phenomenon of overprediction in the calculation of the rotating missile Magnus force [13]. The transition SST model works based on the SST $k-\omega$ model and considers the following two parameters-intermittent factor and local boundary layer momentum thickness-to construct two transport equations. Combining these two transport equations and relevant empirical formulas with the SST $k$ - $\omega$ turbulence model, the four-equation transition SST model can be established. The turbulence model is more sensitive to factors affecting transition, such as turbulence intensity, separation, and pressure gradient, and is more accurate than other turbulence models in the calculation of the rotational missile Magnus force [13].

2.2.1. Intermittent Factor Transport Equation. The transition in a boundary layer and the vortex viscosity coefficient in a
TABLE 1: Variation of parameters with altitude.

\begin{tabular}{lccc}
\hline Altitude $(\mathrm{km})$ & Temperature $(\mathrm{K})$ & Pressure $(\mathrm{Pa})$ & $\mu \times 10^{-5}$ \\
\hline 0 & 289.10 & 101325 & 1.7940 \\
2 & 276.44 & 78535 & 1.7323 \\
4 & 263.79 & 60990 & 1.6694 \\
6 & 251.13 & 46780 & 1.6050 \\
8 & 238.48 & 35391 & 1.5391 \\
10 & 226.15 & 26370 & 1.4733 \\
12 & 221.46 & 19415 & 1.4479 \\
14 & 221.50 & 14265 & 1.4481 \\
16 & 221.50 & 10481 & 1.4481 \\
18 & 221.50 & 7700 & 1.4481 \\
20 & 221.50 & 5657 & 1.4481 \\
30 & 226.51 & 1211 & 1.4753 \\
40 & 250.33 & 286 & 1.6008 \\
60 & 247.06 & 21 & 1.5839 \\
\hline
\end{tabular}

turbulence model are mainly controlled by intermittent factors. The intermittent factor transport equation can be expressed as [16]

$$
\frac{\partial(\rho \gamma)}{\partial t}+\frac{\partial\left(\rho u_{j} \gamma\right)}{\partial x_{j}}=\frac{\partial}{\partial x_{j}}\left[\left(\mu+\frac{u_{t}}{\sigma_{\gamma}}\right) \frac{\partial \gamma}{\partial x_{j}}\right]+\mathrm{P}_{\gamma}-D_{\gamma},
$$

where $\rho$ is density, $t$ is time, $\mu$ is the laminar viscosity coefficient, $\mu_{t}$ is the turbulent viscosity coefficient, $u_{j}$ is speed, $x_{j}$ is the coordinate, $P_{\gamma}$ is the generated term, $D_{\gamma}$ is the dissipative term, and $\gamma$ is the intermittent factor.

2.2.2. Reynolds Number Transport Equation of Transition Momentum Thickness. The Reynolds number of transition momentum thickness is a decisive factor for the transition starting point, and the corresponding transport equation can be expressed as [16]

$$
\frac{\partial\left(\rho \tilde{\mathrm{R}} \mathrm{e}_{\theta t}\right)}{\partial t}+\frac{\partial\left(\rho u_{j} \tilde{\mathrm{R}} \mathrm{e}_{\theta t}\right)}{\partial x_{j}}=P_{\theta t}+\frac{\partial}{\partial x_{j}}\left[\sigma_{\theta t}\left(\mu+\mu_{t}\right) \frac{\partial \tilde{\mathrm{R}} \mathrm{e}_{\theta t}}{\partial x_{j}}\right]
$$

where $\tilde{\mathrm{R}} \mathrm{e}_{\theta_{t}}$ is the Reynolds number of local transition momentum thickness, $P_{\theta t}$ is the generated term, and $\sigma_{\theta t}$ is the diffusion term coefficient.

The generated term of the transport equation can be further written as

$$
P_{\theta t}=c_{\theta t} \frac{\rho}{t}\left(\operatorname{Re}_{\theta t}-\tilde{\operatorname{Re}} e_{\theta t}\right)\left(1.0-F_{\theta t}\right)
$$

where $c_{\theta t}$ is a constant, $F_{\theta t}$ is the switching function, and its value ranges from 1 to 0 from the inside to outside of a boundary layer, and $\operatorname{Re}_{\theta t}$ is the local transition Reynolds 


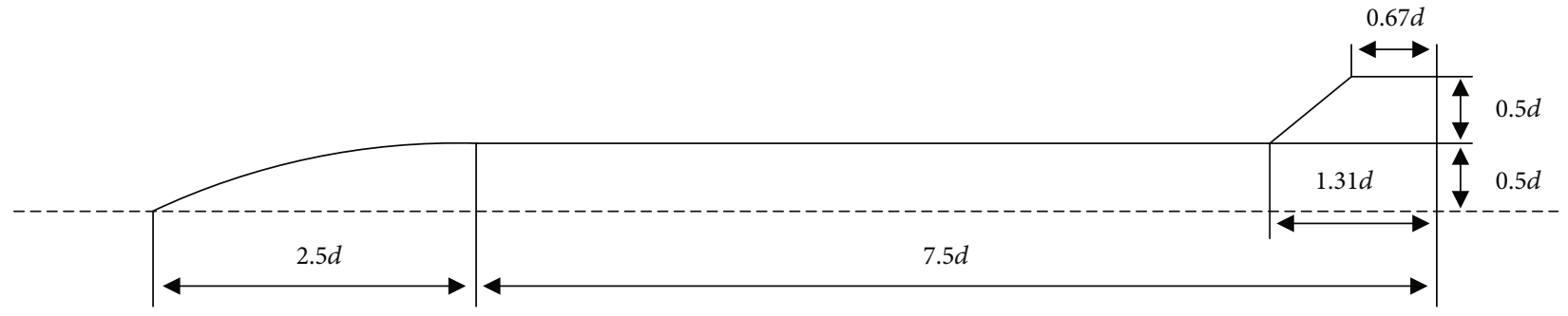

FIgURE 1: Geometric model of the finner.

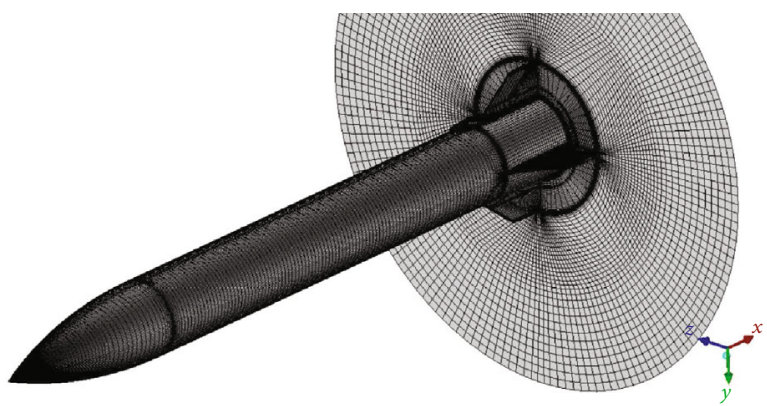

FIGURE 2: Schematic diagram of slip grids.

number, and its value is obtained by fitting flow turbulivity $(\mathrm{Tu})$ and the pressure gradient parameter " $\lambda_{\theta}$ " [17].

$$
\begin{cases}\operatorname{Re}_{\theta t}= \begin{cases}\left(1173.51-589.428 \mathrm{Tu}+\frac{0.2196}{\mathrm{Tu}^{2}}\right) F\left(\lambda_{\theta}\right), & \mathrm{Tu} \leq 1.3, \\ 331.5(\mathrm{Tu}-0.5658)^{-0.671} F\left(\lambda_{\theta}\right), & \mathrm{Tu}>1.3,\end{cases} \\ F\left(\lambda_{\theta}\right)= \begin{cases}1-\left(-12.986 \lambda_{\theta}-123.66 \lambda_{\theta}^{2}-405.689 \lambda_{\theta}^{3}\right) \mathrm{e}^{-(\mathrm{Tu} / 1.5)}, & \lambda_{\theta} \leq 0, \\ 1+0.275\left(1-e^{-35.6 \lambda_{\theta}}\right) e^{-(\mathrm{Tu} / 0.5),} & \lambda_{\theta}>0 .\end{cases} \end{cases}
$$

2.2.3. SST $k$ - $\omega$ Turbulence Model. The transport equations of the SST $k$ - $\omega$ turbulence model are presented below [18].

$$
\frac{\partial(\rho k)}{\partial k}+\frac{\partial\left(\rho U_{j} k\right)}{\partial x_{j}}=\tilde{P}_{k}+\beta^{*} \rho \omega+\frac{\partial}{\partial x_{j}}\left[\left(\mu+\sigma_{k} \mu_{k}\right) \frac{\partial k}{\partial x_{j}}\right],
$$

$$
\begin{aligned}
\frac{\partial(\rho \omega)}{\partial t}+\frac{\partial\left(\rho U_{j} \omega\right)}{\partial x_{j}}= & \frac{\gamma}{\mu_{t}}-\beta \rho \omega^{2}+\frac{\partial}{\partial x_{j}}\left[\left(\mu+\sigma_{\omega} \mu_{t}\right) \frac{\partial \omega}{\partial x_{j}}\right] \\
& +2\left(1-F_{1}\right) \rho \sigma_{\omega 2} \frac{1}{\omega} \frac{\partial k}{\partial x_{j}} \frac{\partial \omega}{\partial x_{j}}
\end{aligned}
$$

$$
\mu_{t}=a_{1} \frac{k}{\max \left(a_{1} \omega b_{1} F_{2} S\right)}
$$

where $U$ is speed, $\tilde{P}$ is the generated term, $k$ is the turbulent kinetic energy, $\omega$ is the dissipation rate of the turbulent kinetic energy ratio, $\sigma_{k}, \sigma_{\omega}, \gamma, a_{1}, \beta^{*}$, and $\beta$ are constants, and $F_{1}$ and $F_{2}$ are the weighting functions of control constants.

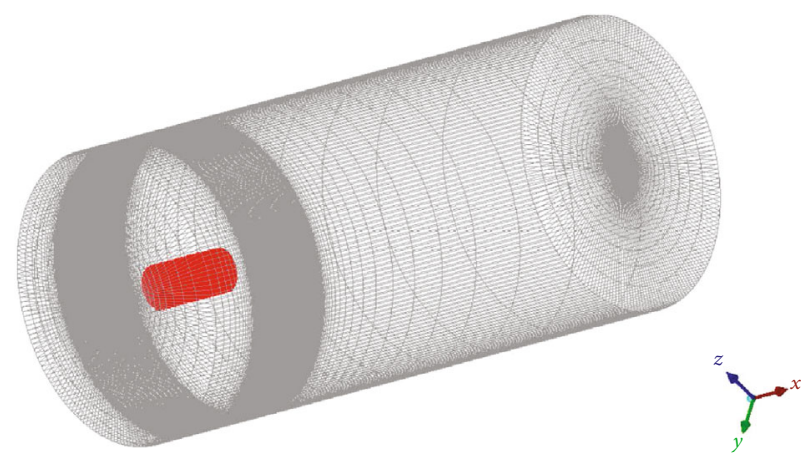

FIGURE 3: Overall grid diagram.

TABLE 2: Grid independence verification.

\begin{tabular}{lcc}
\hline Number of grids & $C_{z}$ & $C_{m y}$ \\
\hline $2.89 \mathrm{M}$ & 0.00872 & -0.19414 \\
$4.52 \mathrm{M}$ & 0.04035 & -0.17457 \\
$7.86 \mathrm{M}$ & 0.03957 & -0.17231 \\
$12.20 \mathrm{M}$ & 0.04002 & -0.17187 \\
\hline
\end{tabular}

2.3. Grid Division and Independence Verification. Threedimensional (3D) structured hexahedral grids are displayed in Figure 2. Under supersonic conditions, the disturbance could only travel downstream within the Mach cone; thus, the upstream flow field was not affected by the disturbance wave. In order to save computing resources, the supersonic far-field grid was extended forward by one time the missile length, backward by 10 times the missile length, and radially by about 20 times the missile radius. Under subsonic conditions, the influence region of the disturbance was the full flow field. Therefore, the subsonic far-field mesh was extended forward by 10 times the missile length, backward by 10 times the missile length, and radially by about 20 times the missile diameter. The altitude of the first layer grid was set as $1.8 \times 10^{-6} \mathrm{~m}$ to maintain $y^{+}<1$. The grid inner boundary was set as a noslip adiabatic wall. Pressure far-field boundary conditions were applied in the current simulation. The turbulent intensity and viscosity of free flow were $0.8 \%$ and 1 , respectively.

The rotation of the missile was realized by the grid movement of the sliding grid area (Figure 3). The sliding grid technology divided the computational domain into two or more elemental regions. During the calculation, these cell regions slid along the mesh interface, the grid of each region did not change, and the data was exchanged between region boundaries. From the warhead to the tail of the missile, the 


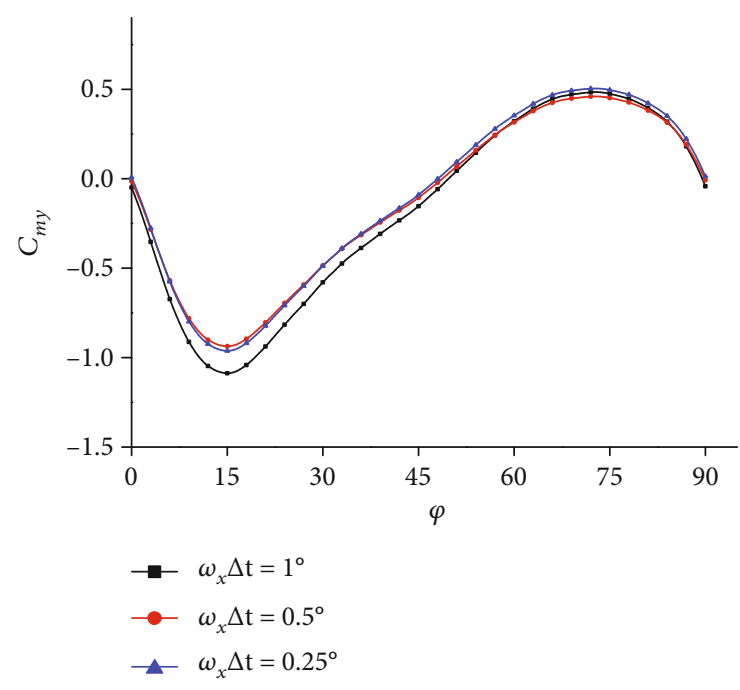

FIgURE 4: Time step independence verification.

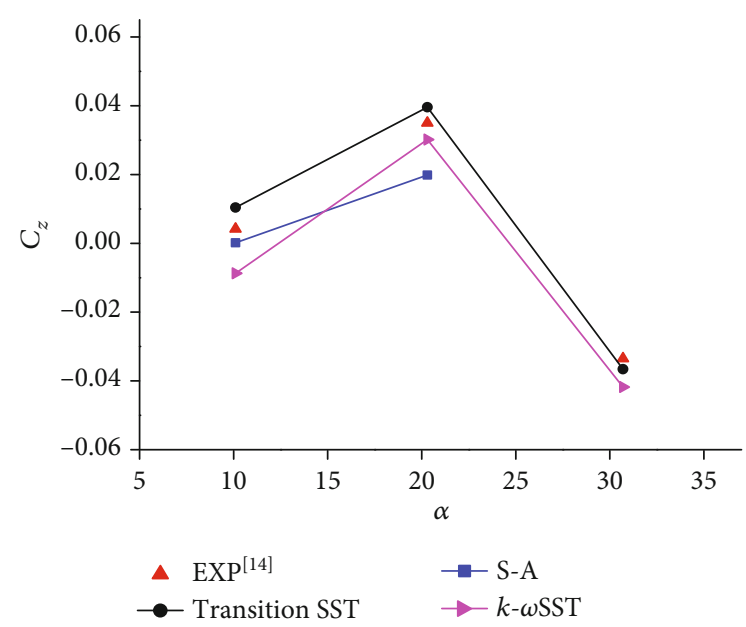

FIGURE 5: Lateral force coefficient.

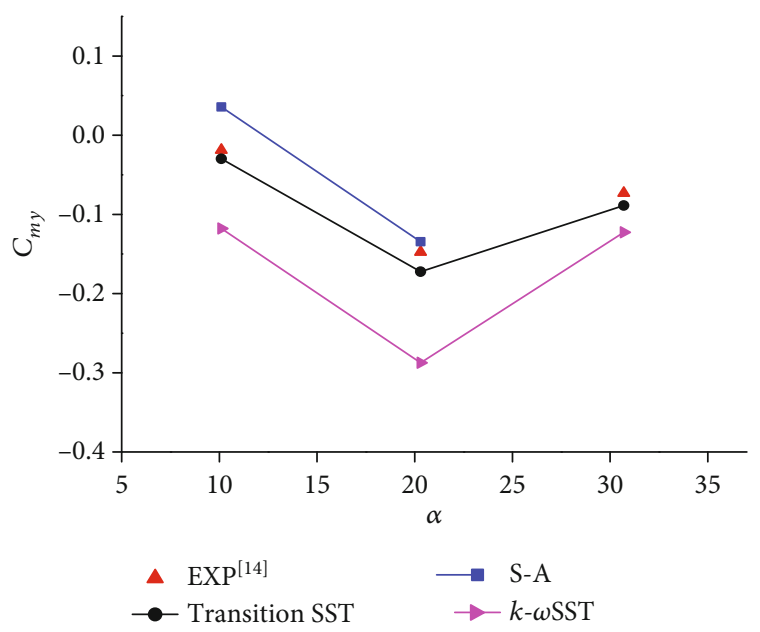

FIgURE 6: Lateral moment coefficient.

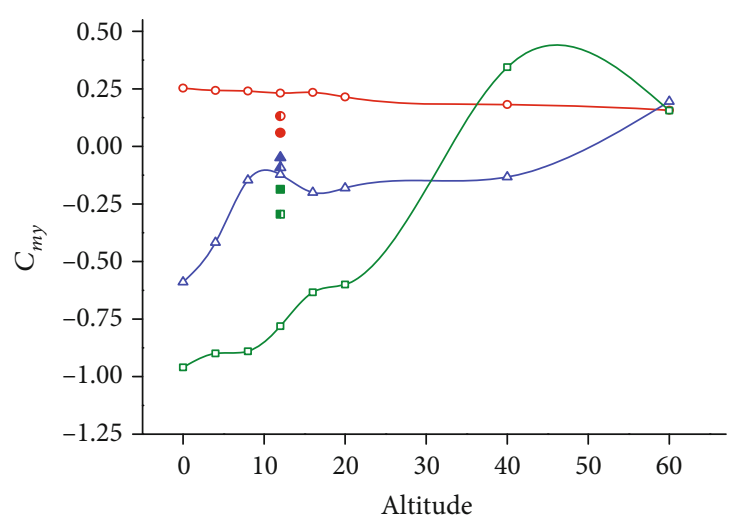

- $a=10^{\circ}$ (Wind tunnel test conditions)

$\triangle \quad a=20^{\circ}$ (Wind tunnel test conditions)

- $a=30^{\circ}$ (Wind tunnel test conditions)

$\multimap-a=10^{\circ}$ (Atmospheric conditions)

$\triangle-a=20^{\circ}$ (Atmospheric conditions)

$\rightarrow-a=30^{\circ}$ (Atmospheric conditions)

- Wind tunnel test value ${ }^{[9]}$

- - Wind tunnel test value ${ }^{[9]}$

- - Wind tunnel test value ${ }^{[9]}$

FIgURE 7: Variation curves of the Magnus moment coefficient with altitude at $\mathrm{Ma}=1.3$ and $p=0.02$.

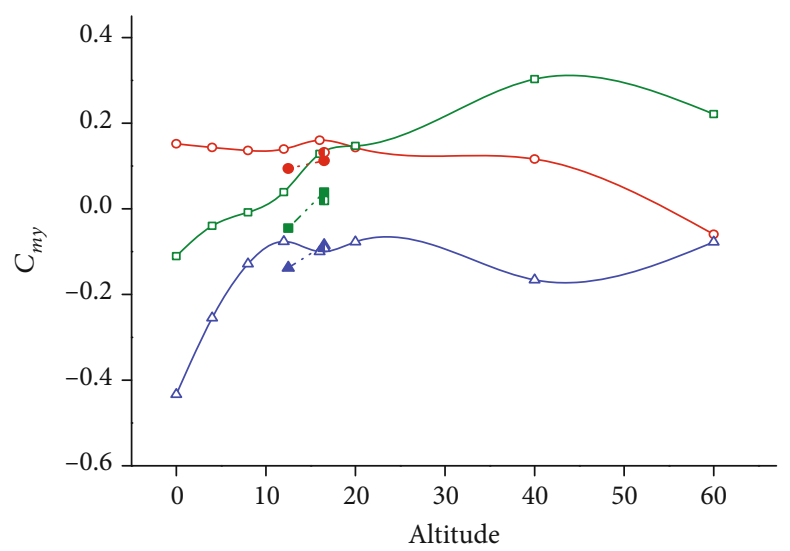

$$
\begin{aligned}
& \text { - } a=10^{\circ} \text { (Wind tunnel test conditions) } \\
& \triangle \quad a=20^{\circ} \text { (Wind tunnel test conditions) } \\
& \text { - } a=30^{\circ} \text { (Wind tunnel test conditions) } \\
& \multimap-a=10^{\circ} \text { (Atmospheric conditions) } \\
& \triangle-a=20^{\circ} \text { (Atmospheric conditions) } \\
& \rightarrow-a=30^{\circ} \text { (Atmospheric conditions) } \\
& \rightarrow \text { Wind tunnel test value } \mathrm{e}^{[9]} \\
& \text { - }- \text { Wind tunnel test value }{ }^{[9]} \\
& \text { - }- \text { Wind tunnel test value }{ }^{[9]}
\end{aligned}
$$

FIgURE 8: Variation curves of the Magnus moment coefficient with altitude at $\mathrm{Ma}=1.5$ and $p=0.02$.

$x$-axis is positive, the XY plane is the angle of attack plane, and the $z$-axis is determined by the right-hand rule. The positive directions of the $z$ - and $y$-axes denote the directions of the lateral force coefficient $\left(C_{z}\right)$ and the lateral moment coefficient $\left(C_{m y}\right)$, respectively. The pitching moment coefficient 


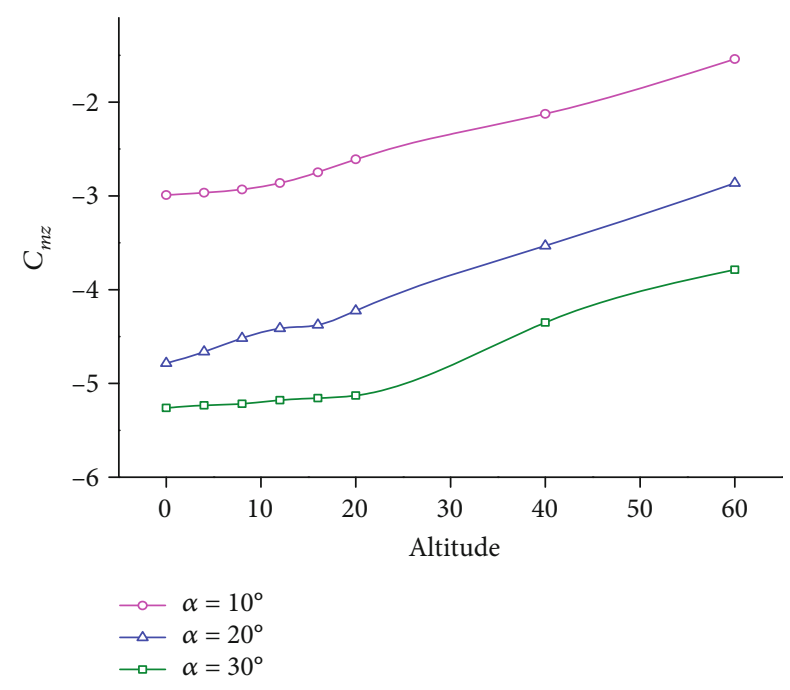

FIGURE 9: Variation curves of the pitching moment coefficient with altitude at $\mathrm{Ma}=1.3$ and $p=0.02$.

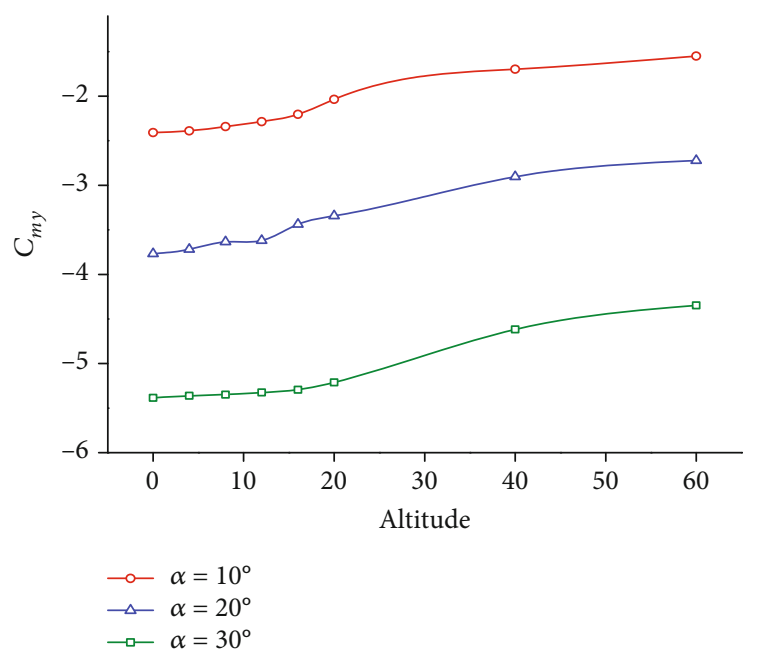

FIGURE 10: Variation curves of the pitching moment coefficient with altitude at $\mathrm{Ma}=1.5$ and $p=0.02$.

$\left(C_{m z}\right)$ direction is negative to the $z$-axis. When viewed from the warhead to the tail, the missile rotates clockwise with a positive spin angle.

The lateral force coefficient and lateral moment coefficient of the missile under different mesh quantities with the Mach number of 1.5 were calculated in Table 2 . When the number of meshes was too small, calculation results of the lateral force coefficient and the lateral moment coefficient were inaccurate. In order to save computing resources and ensure calculation accuracy, 7.8 million and 10.2 million grids (after expanding the front field) were selected under supersonic and subsonic conditions. Three groups of time steps $\left(\omega_{x} \Delta t=0.25^{\circ}, 0.50^{\circ}\right.$, and $\left.1.00^{\circ}\right)$ were selected for time step independence verification; it indicates that each calculated step rotated the missile by $0.25^{\circ}, 0.50^{\circ}$, and $1.00^{\circ}$. Figure 4 displays the variation curve of the lateral force coefficient with the spin angle under three different time steps. It is clear that with the decrease of the time step,

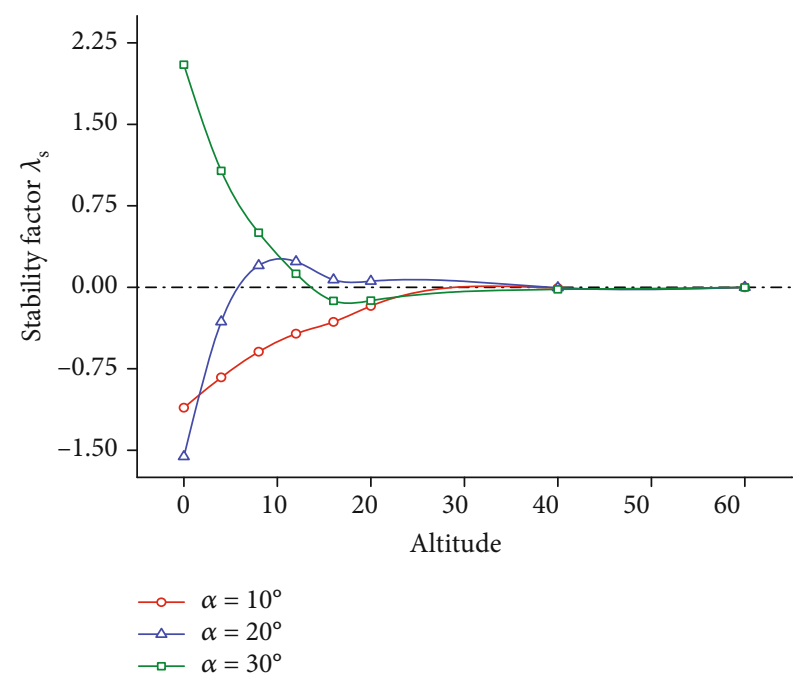

FIgURe 11: Variation curves of the stability factor with altitude at $\mathrm{Ma}=1.5$ and $p=0.02$.

the calculation results of $\omega_{x} \Delta t=0.25^{\circ}$ and $\omega_{x} \Delta t=0.50^{\circ}$ gradually approached each other, whereas a certain gap existed between the results of $\omega_{x} \Delta t=0.50^{\circ}$ and $\omega_{x} \Delta t=1.00^{\circ}$. In order to save computing resources, the time step was set to $\omega_{x} \Delta t=0.50^{\circ}$.

2.4. Validation of the Numerical Method. The lateral force coefficient and lateral moment coefficient of the missile under different turbulence models are exhibited in Figures 5 and 6, respectively. The following experimental conditions were selected for the validation of the proposed numerical method: Mach number $\mathrm{Ma}=1.5$, Reynolds number (based on the model diameter) $\operatorname{Re}=4.13 \times 10^{5}$, total pressure $P_{0}=65374$ $\mathrm{Pa}$, total temperature $T_{0}=311 \mathrm{~K}$, angle of attack $\alpha=10.1^{\circ}$, $20.3^{\circ}$, and $30.7^{\circ}$, and nondimensional spin rate $p=\omega_{x} d /(2$ $\left.V_{\infty}\right)=0.02$. The corresponding freestream conditions were $P_{\infty}=17808.1 \mathrm{~Pa}, T_{\infty}=214.48 \mathrm{~K}, V_{\infty}=440 \mathrm{~m} / \mathrm{s}$, and spin rate $\omega_{x}=385.25 \mathrm{rad} / \mathrm{s}$, respectively.

It is evident that the calculated results of the transition SST model were close to the experimental values. Therefore, the adoption of the transition SST model has a certain credibility.

\section{Stability Analysis of Rotating Missiles}

For rotating missiles with tail fins, the dynamic stability factor $\left(S_{d}\right)$ can be expressed as [12]

$$
S_{d}=\frac{\left(C_{L \alpha} / 2 m\right)+\left(d^{2} C_{M_{p \alpha}} / 2 I_{x}\right)}{\left(C_{L \alpha} / 2 m\right)-\left(d^{2} C_{M_{q}} / 2 I_{y}\right)},
$$

where $C_{L \alpha}$ is the lift coefficient, $C_{M p \alpha}$ is the Magnus moment coefficient, and $C_{M q}$ is the damping moment coefficient of pitch. 


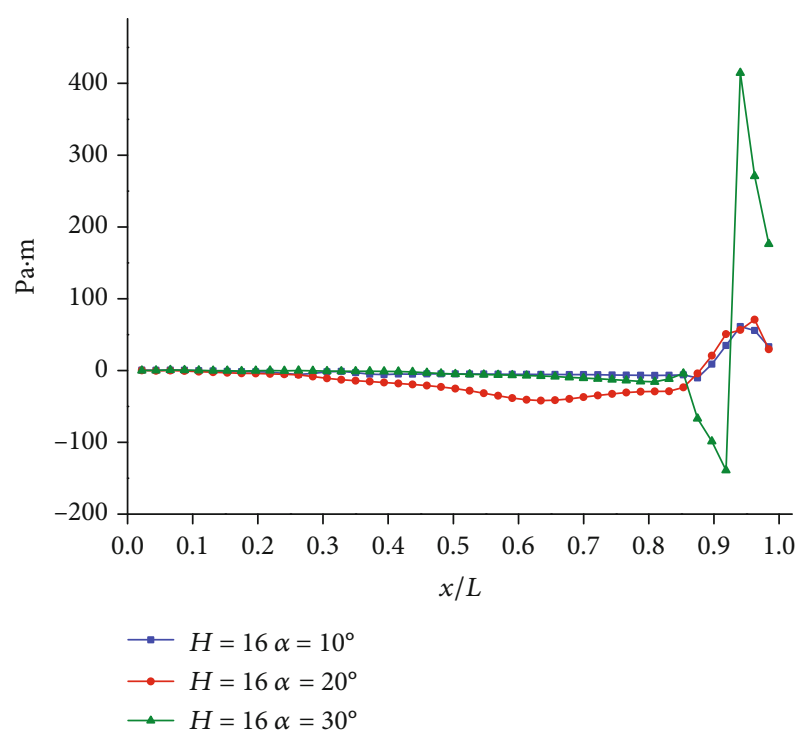

FIGURE 12: Variations of the average lateral pressure difference of the missile body along the missile axis at $\mathrm{Ma}=1.5$ and $p=0.02$.

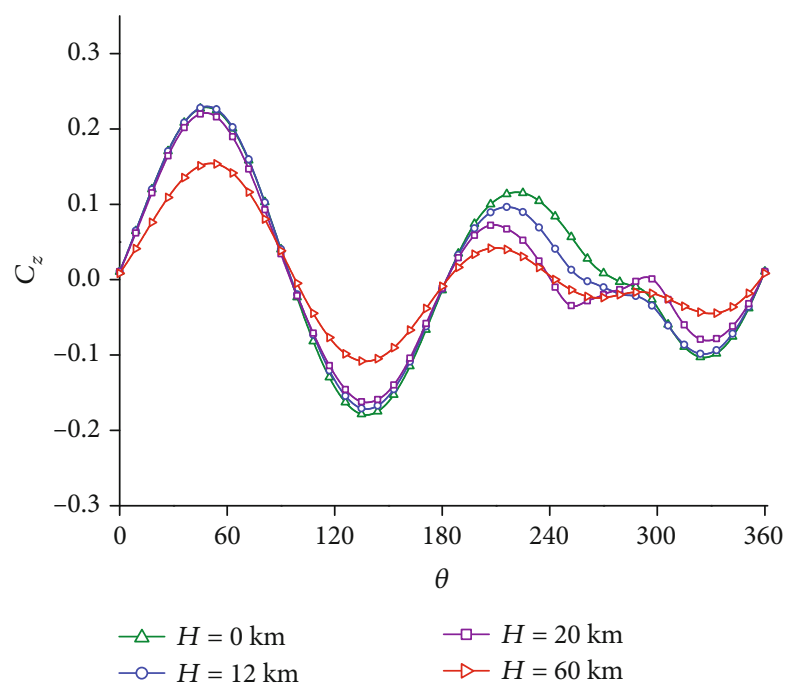

FIGURE 13: Lateral force coefficient curves of the single tail fin at $\mathrm{Ma}=1.5$ and $p=0.02$.

The dynamic stability condition of a rotating missile with a tail fin can be formulated as

$$
\frac{4 M}{p^{2}}<S_{d}\left(2-S_{d}\right)
$$

where $P=\left(I_{x} / I_{y}\right)(p d / V), M=\rho S d^{3} C_{M_{\alpha}} / 2 I_{y}, C_{M \alpha}$ is the pitching moment coefficient, and $p$ is rotating speed.

When $0<S_{d}<2$, as long as the missile remains statically stable (pitch moment coefficient $C_{M \alpha}<0$ ), it must also be dynamically stable.

For axisymmetric missiles, $C_{L \alpha}>0$ and $C_{M q}<0$. The direction of the Magnus torque tends to change during the transonic phase; thus, at this time, $C_{M p \alpha}<0$ and $S_{d}<0$.

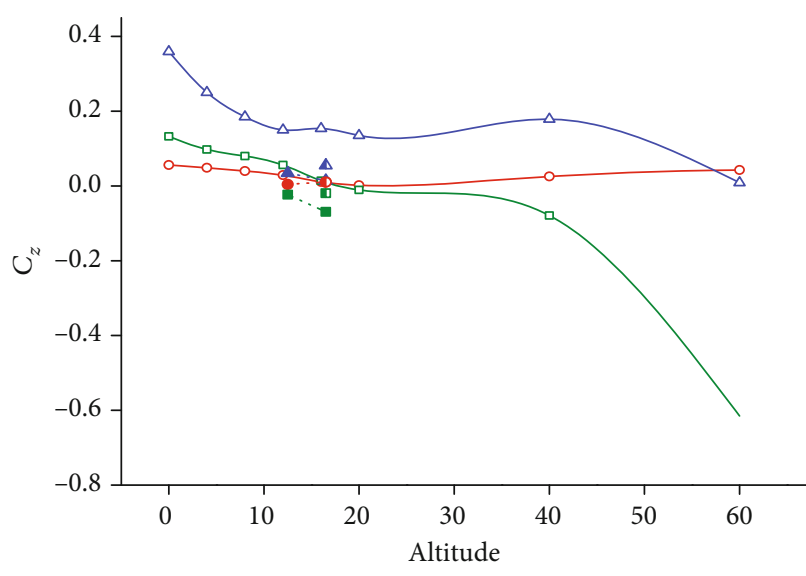

$$
\begin{aligned}
& -\circ \quad a=10^{\circ} \text { (Atmospheric conditions) } \\
& -\triangle a=20^{\circ} \text { (Atmospheric conditions) } \\
& \rightarrow-a=30^{\circ} \text { (Atmospheric conditions) } \\
& \text { - } a=10^{\circ} \text { (Wind tunnel test conditions) } \\
& \Delta \quad a=20^{\circ} \text { (Wind tunnel test conditions) } \\
& \text { - } a=30^{\circ} \text { (Wind tunnel test conditions) } \\
& \text { - Wind tunnel test value }{ }^{[9]} \\
& \text { ^- Wind tunnel test value }{ }^{[9]} \\
& - \text { Wind tunnel test value }{ }^{[9]}
\end{aligned}
$$

FIgURE 14: Variation curves of the Magnus force coefficient with altitude at $\mathrm{Ma}=1.5$ and $p=0.02$.

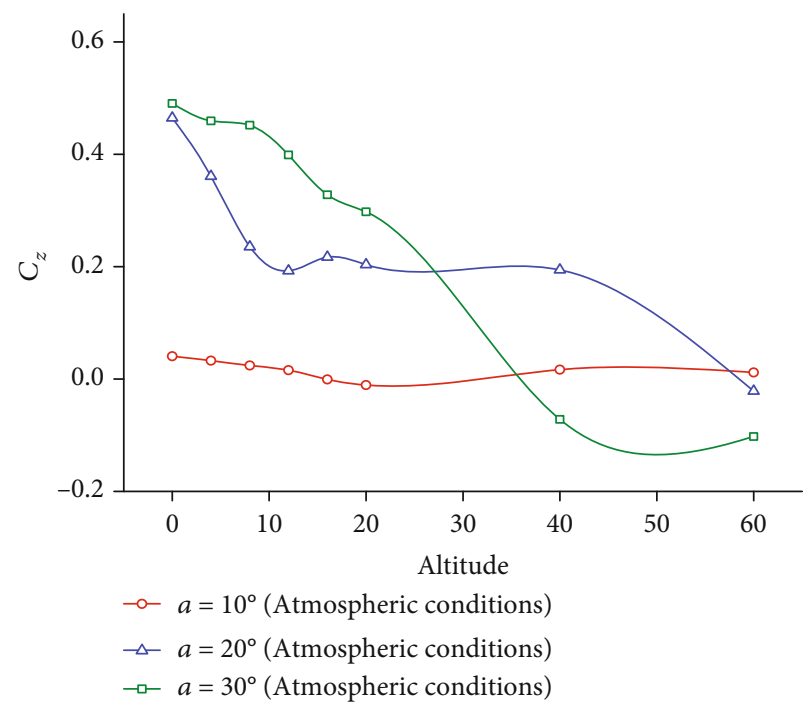

FIgURE 15: Variation curves of the Magnus force coefficient with altitude at $\mathrm{Ma}=1.3$ and $p=0.02$.

When $S_{d}<0$, the dynamic stability condition of a rotating missile with a tail fin can be expressed as [12]

$$
P^{2}<\frac{4 M}{S_{d}\left(2-S_{d}\right)}
$$

Generally, three variables determine the stability of a rotating missile-pitch moment coefficient $\left(C_{M \alpha}\right)$, rotating speed $(p)$, and dynamic stability factor $\left(S_{d}\right)$. The absolute 


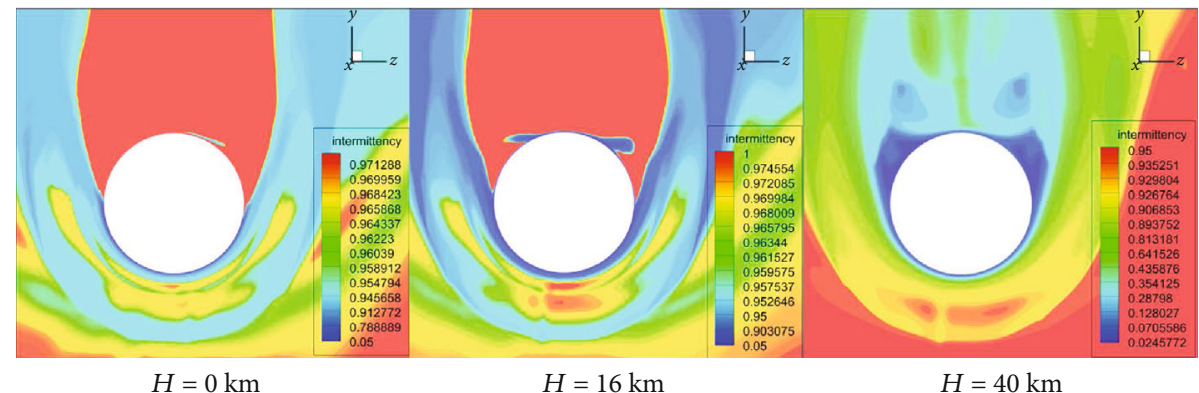

Figure 16: Cloud maps of the intermittent factor at $x=0.36$ cross section of the missile body and an angle of attack of $30^{\circ}$.

value of $C_{M \alpha}$ decreases, whereas those of $S_{d}$ and $p$ increase. These three factors, whether acting alone or together, may lead to a failure of inequality 10. At this point, the missile cannot satisfy the dynamic stability condition. Under a low upper-air density, if the absolute value of $C_{M \alpha}$ decreases and $p$ is too high, dynamic instability will occur. Zhang et al. [11] conducted telemetry on a missile at the plateau and found that under a large angle of attack, the missile had a large attack angle cone pendulum movement and the attack angle reached greater than $30^{\circ}$.

Theoretically, the following situation may occur. When a missile is launched at a high angle of launch, transonic conditions tend to occur at the highest point of its trajectory [11]. With the increasing altitude, the center of pressure of the missile moves forward, the static stability decreases, and the angle of attack increases. The Magnus torque coefficient becomes negative due to the low upper-air density. When the static stability is reduced and the missile does not meet the dynamic stability condition, the attack angle further increases. However, with the increase of the angle of attack, the Magnus moment coefficient of the missile changes from negative to positive; consequently, the dynamic stability condition is satisfied. Therefore, it is important to understand how the Magnus moment varies with altitude.

\section{Analysis of Magnus Torque Characteristics}

\subsection{Variation of the Magnus Moment with Altitude. Figures 7} and 8 display the variations of the time-averaged Magnus moment coefficient with altitude for $\mathrm{Ma}=1.3$ and 1.5, respectively. The experimental values in the figure refer to wind tunnel test values corresponding to the same Reynolds numbers at different altitudes, indicating that the Reynolds numbers of the missile at different altitudes were similar to those of the wind tunnel test. The variations of the Magnus moment coefficient with altitude at different angles of attack were also similar to those of the wind tunnel test. The difference between simulated and experimental values under atmospheric conditions occurred due to the difference between wind tunnel test and atmospheric conditions. Simulation results under wind tunnel test conditions were in good agreement with experimental values. With the increase of the angle of attack, the direction of the Magnus moment coefficient changed, especially at high altitudes, the Magnus moment coefficient first changed from positive to negative and then from negative to positive.

Figures 9 and 10 present the variation curves of the missile pitching moment with altitude. With the increasing altitude, the absolute value of the missile pitch moment coefficient decreased and the pressure center moved forward; hence, the static stability of the missile decreased; consequently, the equilibrium angle of attack increased. However, the phenomenon of negative Magnus moment occurred at larger angles of attack; thus, the missile did not meet the dynamic stability condition, and its angle of attack further increased. When the missile flew under the conditions of $\mathrm{Ma}=1.3$ and $H=16 \mathrm{~km}$, with the increase of the angle of attack, the Magnus moment was always negative, causing a divergence of the conical pendulum. When the missile flew under the conditions of $\mathrm{Ma}=1.5$ and $H=16 \mathrm{~km}$, with the increase of the angle of attack, the Magnus moment changed from negative to positive and the missile met the dynamic stability condition; thus, it experienced a large attack angle cone pendulum movement. The variation of the missile stability factor $\left(\lambda_{s}\right)$ with altitude is exhibited in Figure 11 and further confirms the possibility of the above working conditions. Generally, the stability factor $\lambda_{s}$ is used to analyze the dynamic stability of a missile on an external trajectory. When $\lambda_{s}<0$, the greater the absolute value of $\lambda_{s}$, the faster the convergence speed of flight attack angle to the equilibrium point and the better the dynamic stability. When $\lambda_{s}>0$, a missile becomes unstable [12]. At an angle of attack of $20^{\circ}$, with the increasing altitude, the stability factor changed from negative to positive and the missile gradually became unstable. At an angle of attack of $30^{\circ}$, with the increasing altitude, the stability factor changed from positive to negative and the missile gradually became stable; therefore, it experiences a large attack angle cone pendulum movement.

Two important characteristics were noticed in the variation of the Magnus moment characteristics of the missile. First, the direction of the Magnus moment changed with the increase of the angle of attack. Second, at high angles of attack, the direction of the Magnus moment changed with the increasing altitude.

Figure 12 displays the distribution curve of lateral pressure difference along the missile axis when $\mathrm{Ma}=1.5$ and $H$ $=16 \mathrm{~km}$. It is noticeable that the lateral force direction generated by the missile body was opposite to that generated by the tail fin. However, when the angle of attack was $20^{\circ}$, the lateral 

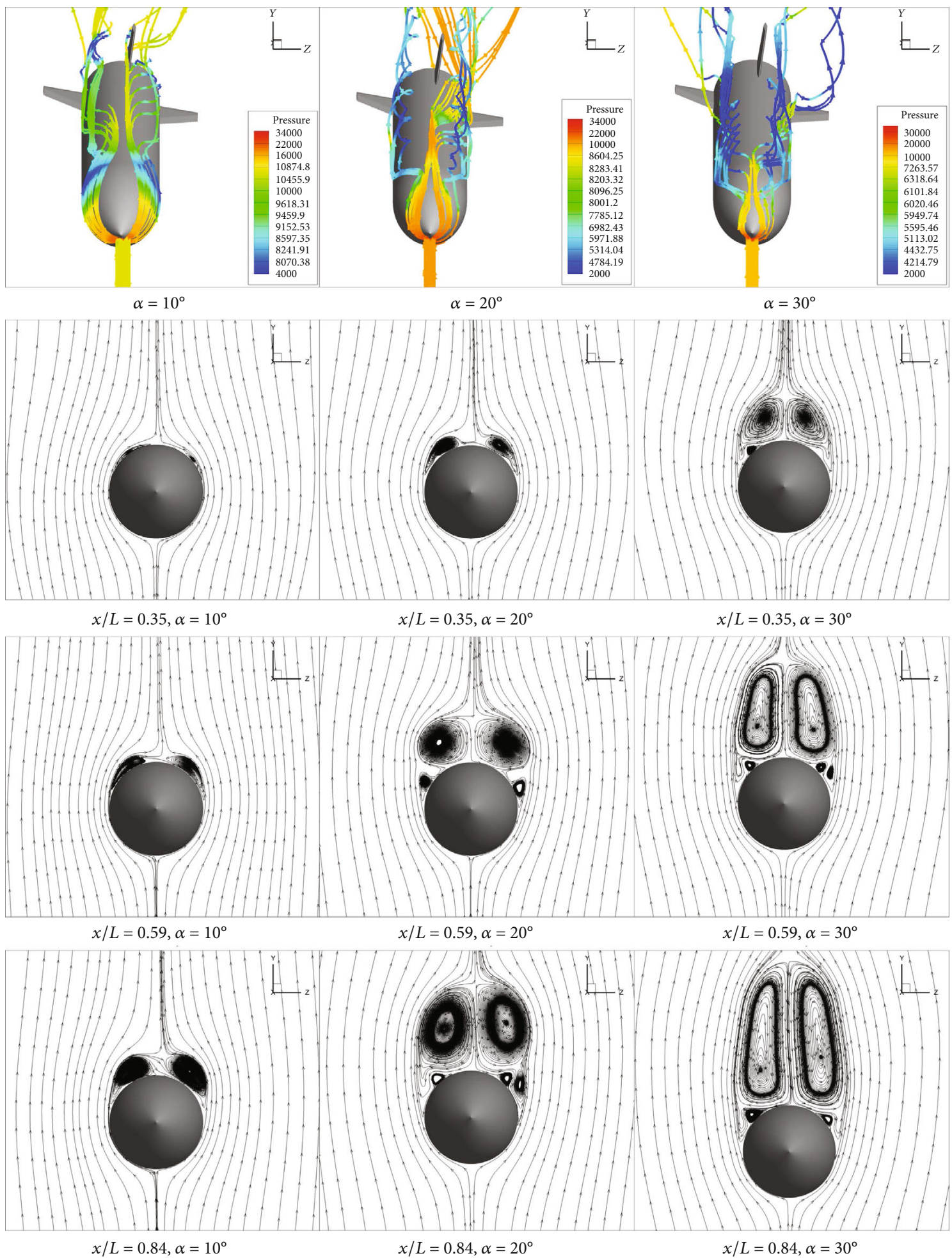

Figure 17: Streamlines at $H=16 \mathrm{~km}$ and $\mathrm{Ma}=1.5$.

force along the missile axis was different from that generated by other angles of attack. The lateral force generated by the latter part of the missile body was larger than that generated by the other two angles of attack. However, the lateral force of the tail fin was similar to that generated by the angle of attack of $10^{\circ}$; thus, the Magnus moment direction changed with the increase of the angle of attack.

Figure 13 displays the variation of the lateral force coefficient curve of the single tail fin. With the increasing altitude, the lateral force coefficient of the tail fin decreased and its 

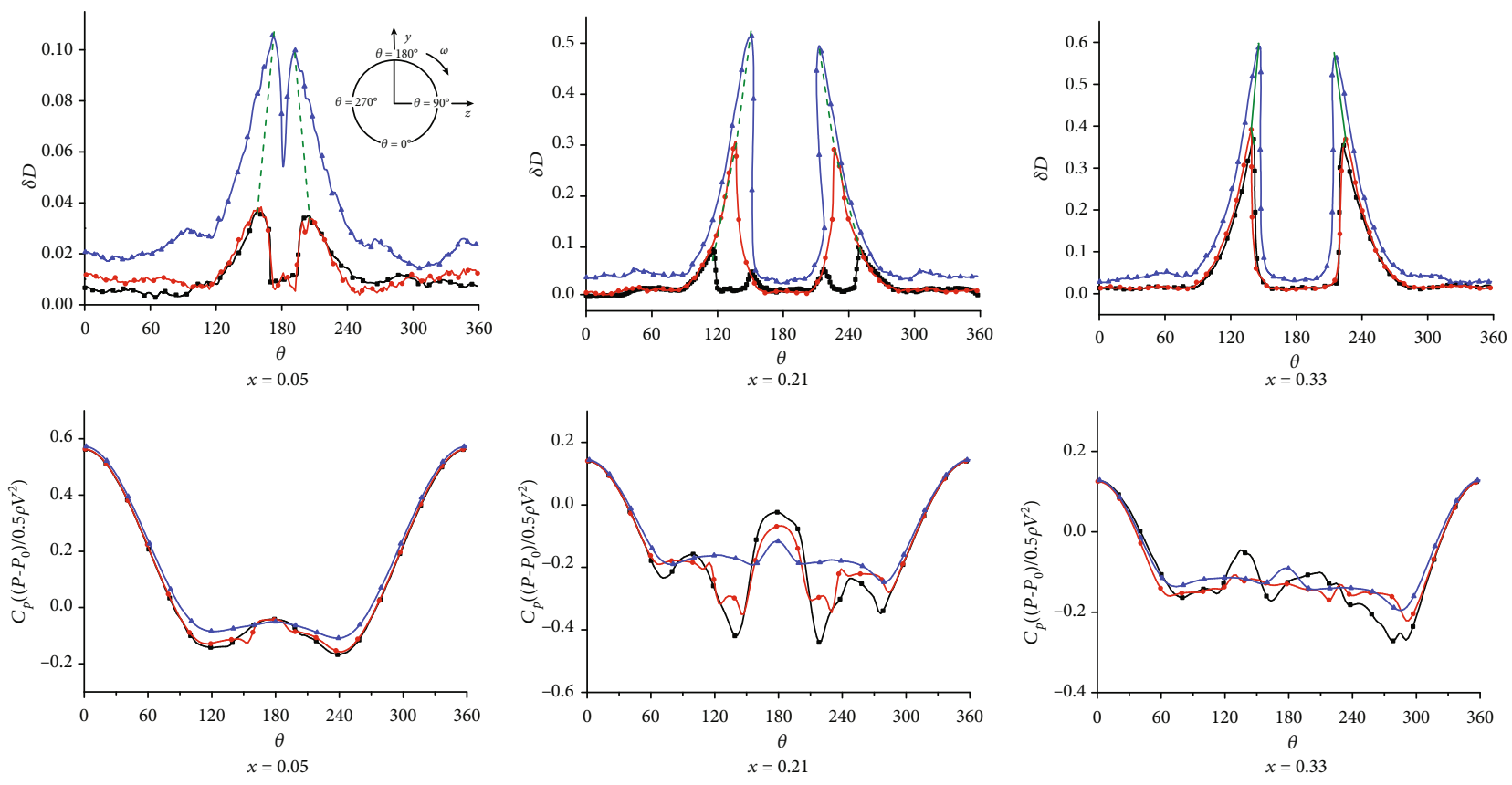

$$
\begin{aligned}
& \because H=0 \mathrm{~km} \\
& \therefore H=16 \mathrm{~km} \\
& \therefore H=40 \mathrm{~km}
\end{aligned}
$$

FIgURE 18: Boundary layer thickness and $C_{p}$ curves at different cross sections when $\mathrm{Ma}=1.5$ and $\alpha=20^{\circ}$.
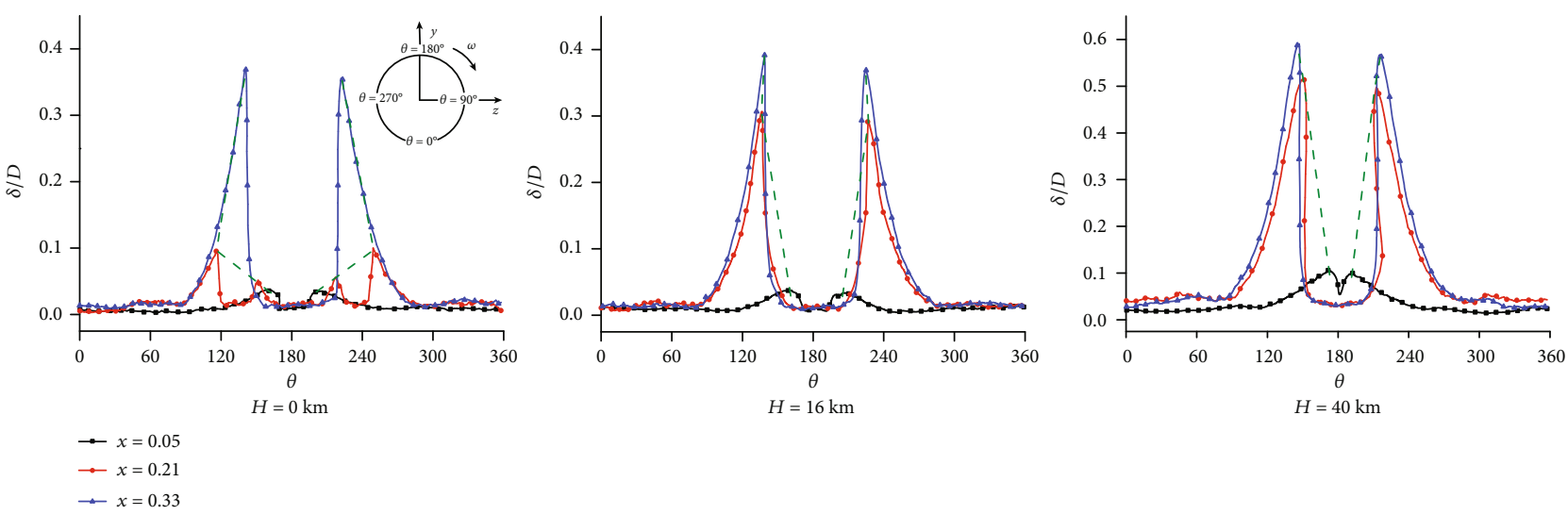

Figure 19: Boundary layer thickness curves at different altitudes when $\mathrm{Ma}=1.5$ and $\alpha=20^{\circ}$.

periodic average decreased from 0.016218 to 0.006778 . As the lateral force of the missile body was opposite to that of the tail fin and the lateral force of the tail fin decreased with the increasing altitude, the direction of the Magnus moment changed with the increasing altitude.

4.2. Variation of the Magnus Force with Altitude. Figures 14 and 15 display the variation curves of the Magnus force with altitude. It is noticeable that the Magnus force direction changed with altitude at an angle of attack of $30^{\circ}$. Figure 16 presents the variations of the intermittent factor $(\gamma)$ cloud with altitude at $x=0.36$ cross section of the missile body and an angle of attack of $30^{\circ}$. The intermittent factor was used to measure the timeshare of turbulence at a point in the transition region. When the intermittent factor was 1 , the flow was completely turbulent, whereas when the inter- mission factor was 0 , the flow was completely laminar. With the increasing altitude, the air density decreased, causing a decrease in the Reynolds number. With the increasing altitude, the upper surface of the missile body changed from a turbulent boundary layer to a laminar one. This phenomenon had a great influence on the flow separation on the missile surface and caused the direction of the Magnus force to change.

Figure 17 presents the streamline diagrams of the missile at different angles of attack when the altitude was $16 \mathrm{~km}$ and the Mach number was 1.5. The colors of the streamline diagrams represent pressures at the streamline. The streamline diagram at the angle of attack of $20^{\circ}$ was different from those of the other two angles of attack. At the angle of attack of $20^{\circ}$, the wash flow generated by the left lower part of the head was partially wash to the right side of the missile body on the 


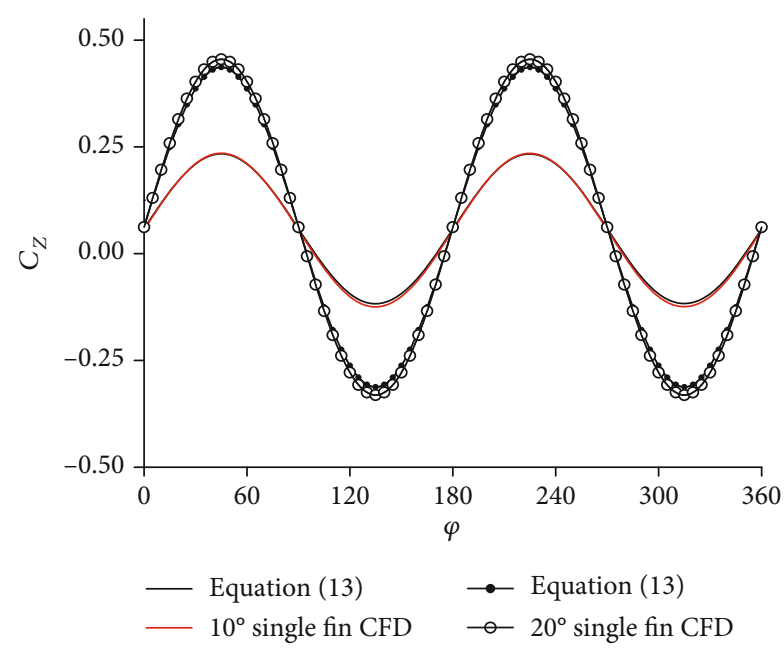

FIGURE 20: Cyclic variation curves of the lateral force coefficient of the single tail fin.

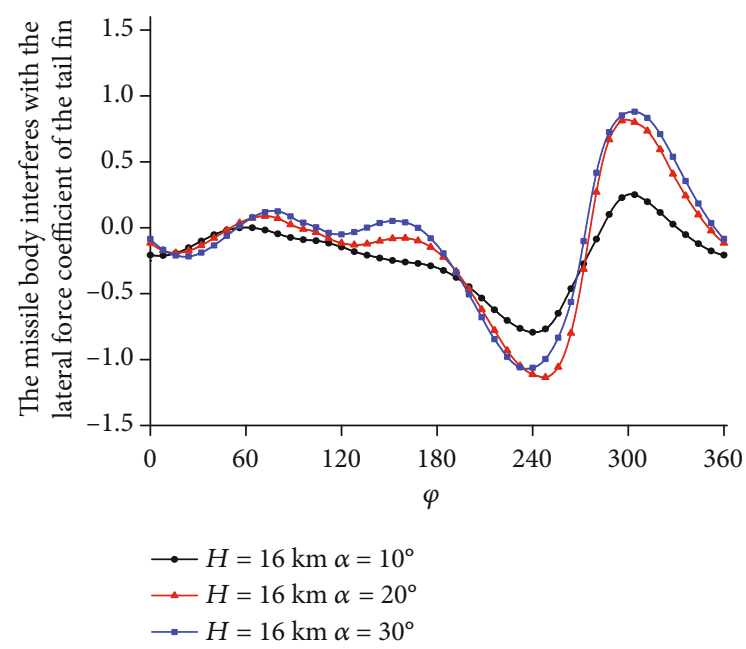

Figure 21: Interference of the missile body with the lateral force coefficient of the tail fin at $\mathrm{Ma}=1.5$.

leeward side. From the colors of the streamline diagrams, it is evident that this part of the wash flow caused a high pressure on the right rear side of the missile body, resulting in the phenomenon shown in Figure 12.

Figures 18 and 19 illustrate the boundary layer thickness and $C_{p}$ curves at different cross sections and altitudes. With the increasing altitude, the boundary layer thickness increased. The asymmetric distortion of the boundary layer was the main cause of the lateral force. It is noticeable from Figure 18 that the thickness peak of the boundary layer on the right side was more serious than that on the leeward side of the missile. When the altitude was low, the boundary layer thickness became larger only in the region near the tail $(x=0.33)$, leading to an asymmetric distortion. The larger the boundary layer thickness, the greater the lateral force intensity produced by the asymmetric distortion; thus, the body behind the center of mass (especially in front of the tail wing) had a larger lateral force and the Magnus moment generated by the missile body became larger. With the increasing altitude, the position with a larger boundary layer thickness gradually moved forward. When the altitude was $16 \mathrm{~km}$, the boundary layer thickness at $x=0.05$ was relatively small; however, the boundary layer thickness at $x=0.21$ was larger. When the altitude was $40 \mathrm{~km}$, the boundary layer thickness of the missile was relatively larger. The forward movement of the thicker boundary layer made the lateral force distribution of the missile body uniform; hence, Magnus moments produced by different thicknesses of front and rear boundary layers of the missile body decreased and the Magnus moment generated by the tail wing increased, causing a change in the Magnus torque direction.

4.3. Interference of the Missile Body with the Lateral Force of the Tail Fin. The normal force was obtained by calculating the effective angle of attack of the rotating tail fin, and the lift force was projected to the $z$-axis to obtain the lateral force generated by the tail fin. The effective angle of attack of the tail fin during rotation was calculated as

$$
\alpha_{e}=\alpha \pm \frac{\omega_{\mathrm{x}} y}{V_{\infty}}
$$

where the former is the angle of attack of the missile and the latter is the additional angle of attack caused by rotation.

Now, using the derivative formula of the normal force coefficient of a quadrangle airfoil proposed by Harmon [19], the normal force of the single tail fin could be expressed as

$$
F_{n}=\int_{d / 2}^{d} q C_{n \alpha}^{\prime} \alpha_{e} c(y) d y=q C_{n \alpha}^{\prime}\left(0.00104 \alpha \pm 3.44 \times \frac{10^{-5} \omega_{x}}{V_{\infty}}\right)
$$

where $c$ is the chord length, $c(y)=C-(y-d / 2) / \tan \theta$, the sweeping angle of the leading edge is $36.9^{\circ}, C_{n \alpha}^{\prime}=4(1-0.5$ $A B) / B, A$ is the aspect ratio of the wing, and $B=$ $\left(\mathrm{Ma}^{2}-1\right)^{1 / 2}$.

Therefore, the lateral force coefficient of the tail fin is

$$
C_{z}=-\cos (\varphi) q C_{n \alpha}^{\prime}\left(\alpha \sin (\varphi)+\frac{\omega_{x}}{V_{\infty}}\right) .
$$

Figure 20 displays the periodic variation curves of the lateral force coefficient of the single tail fin calculated by Equation (13) and CFD simulations at $\mathrm{Ma}=1.5$ and $H=16 \mathrm{~km}$. Calculation results of Equation (13) were relatively consistent with those of CFD simulations; thus, the proposed method could be used to quickly predict the periodic lateral force coefficient of a single tail fin. The ratios of the influence of the missile body on the lateral force coefficient of the tail fin at different angles of attack and altitudes were obtained by subtracting the lateral force coefficient of the tail fin alone and dividing it by the maximum value of the lateral force coefficient of the tail fin. 


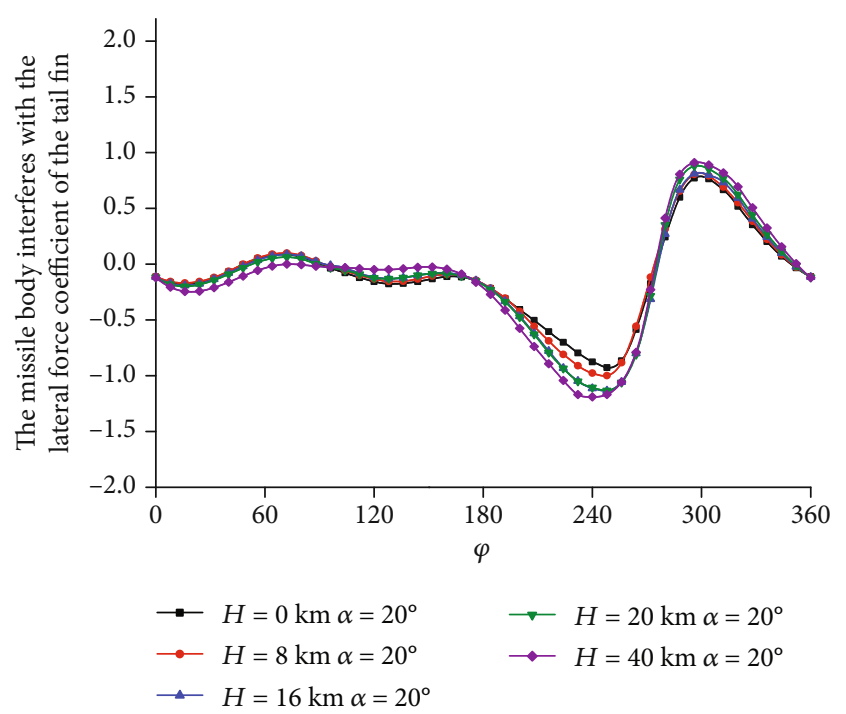

FIGURE 22: Interference of the missile body with the lateral force coefficient of the tail fin at $\mathrm{Ma}=1.5$.

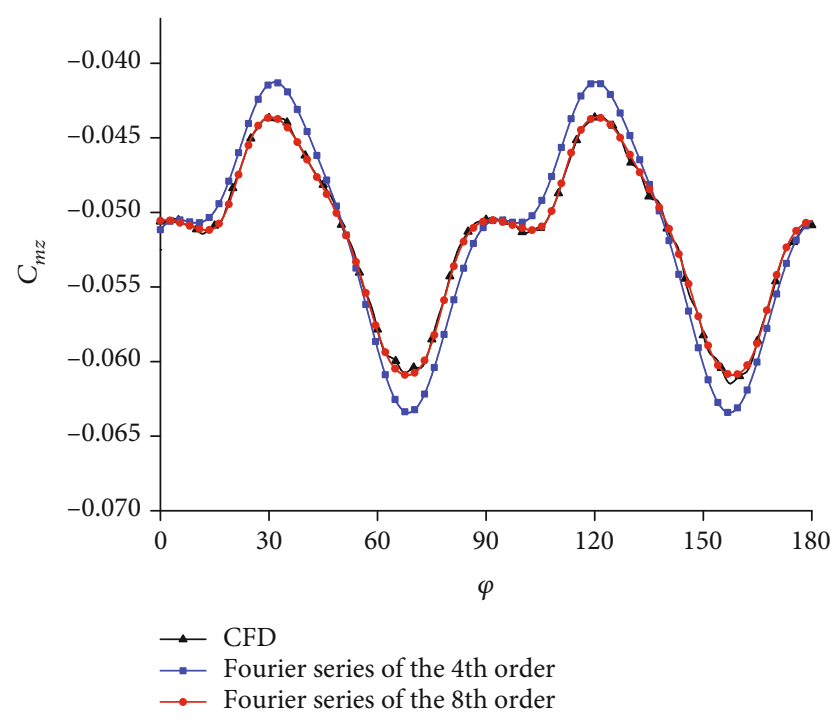

FIGURE 23: Comparison of lateral force coefficient curves obtained by different Fourier series.

Figures 21 and 22, respectively, exhibit the interferences of the missile body with the lateral force coefficient of the tail fin at different altitudes and angles of attack when $\mathrm{Ma}=1.5$. In the leeward area, the missile body had a greater disturbance to the lateral force coefficient of the tail fin. When the angles of attack were $20^{\circ}$ and $30^{\circ}$, the interference of the missile body with the tail fin was greater than that at the angle of attack of $10^{\circ}$. It happened because with the increase of the angle of attack, the shielding of the missile body against the tail affected the aerodynamic characteristics of the tail and the influence was abrupt. It also explains the reason why lateral forces generated by the missile tail fin were similar at the angles of attack of $10^{\circ}$ and $20^{\circ}$ (Figure 12). When the angle of attack is $20^{\circ}$, the interference of the missile body with the tail fin suddenly increased.

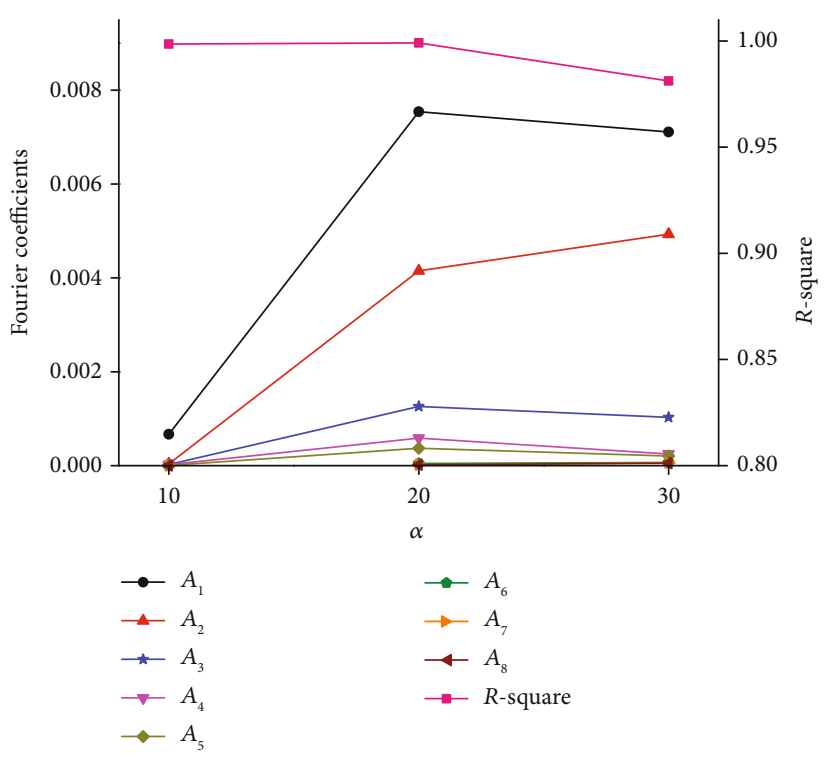

FIgURE 24: Fourier coefficient of the missile body without tail fins.

4.4. Frequency Analysis of the Interference of the Missile Body with the Lateral Force of the Tail Fin. With the increase of the angle of attack, the lateral force characteristics of the missile body changed because of the interference of the tail fin with the missile body. The lateral force coefficient curve of the missile body was fitted by the following Fourier series.

$$
C_{z}=A_{0}+\sum_{n} A_{n} \sin \left(n \omega t+\theta_{n}\right)
$$

where $A_{0}$ represents the time-averaged body Magnus force coefficient and $A_{n}$ and $\theta_{n}$ are the corresponding Fourier coefficient and phase, respectively. Least-squares fitting was adopted to obtain the Fourier coefficients at different angles of attack. $R$-square describes the correlation between response values and predicted response values, and it is defined as the ratio of the sum of squares of regression and the sum of squares about the mean. Figure 23 presents the fitting results of the lateral force coefficient curve of the missile based on the $4^{\text {th- }}$ and 8 th-order Fourier series, and the corresponding $R$-square values were 0.89846 and 0.99815 , respectively. It is clear that the 8 th-order Fourier series can be used for better fitting.

Figure 24 presents the fitting result of the lateral force coefficient curve of the missile body without tail fins based on the 8th-order Fourier series, and it is observable that the amplitudes of $A_{1}-A_{3}$ varied significantly. Figure 25 displays the fitting result of the lateral force coefficient curve of the missile body with tail fins based on the 8th-order Fourier series. In this case, the amplitudes of $A_{1}$ and $A_{3}$ increased with the increase of the angle of attack, whereas that of $A_{2}$ first increased and then decreased with the increase of the angle of attack. The first three Fourier series frequencies, especially $A_{1}$ and $A_{2}$, dominated the fitting curves. The values of $A_{4}-A_{8}$ in Figure 24 are very small. In comparison to Figure 25, the increase of $A_{4}-A_{8}$ in Figure 24 is over $80 \%$. In addition, the $R$-square values in Figure 24 are all greater 


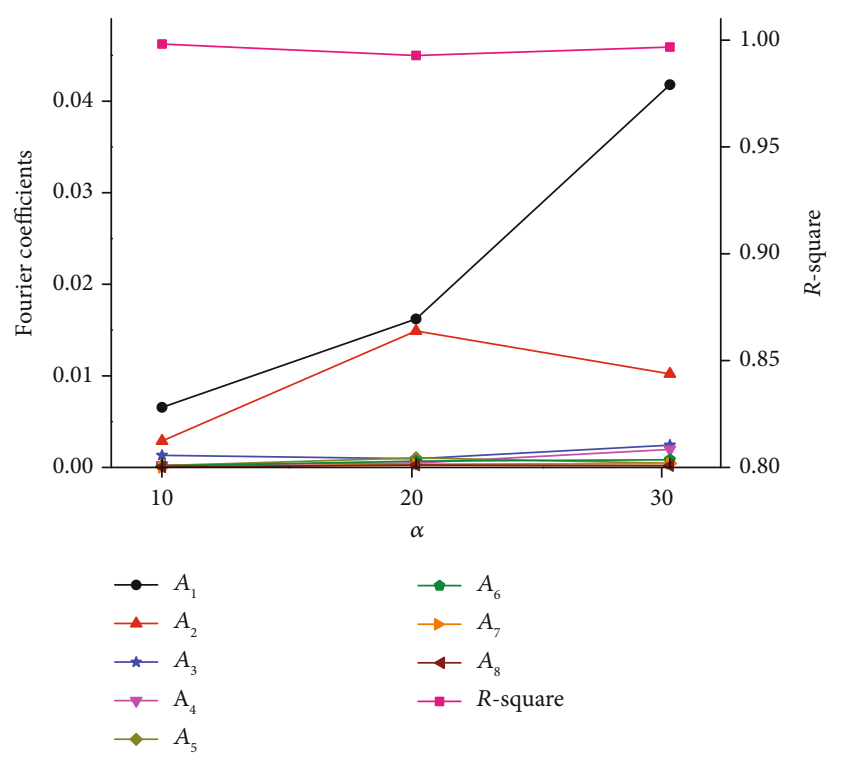

FIGURE 25: Fourier coefficient of the missile body with tail fins.

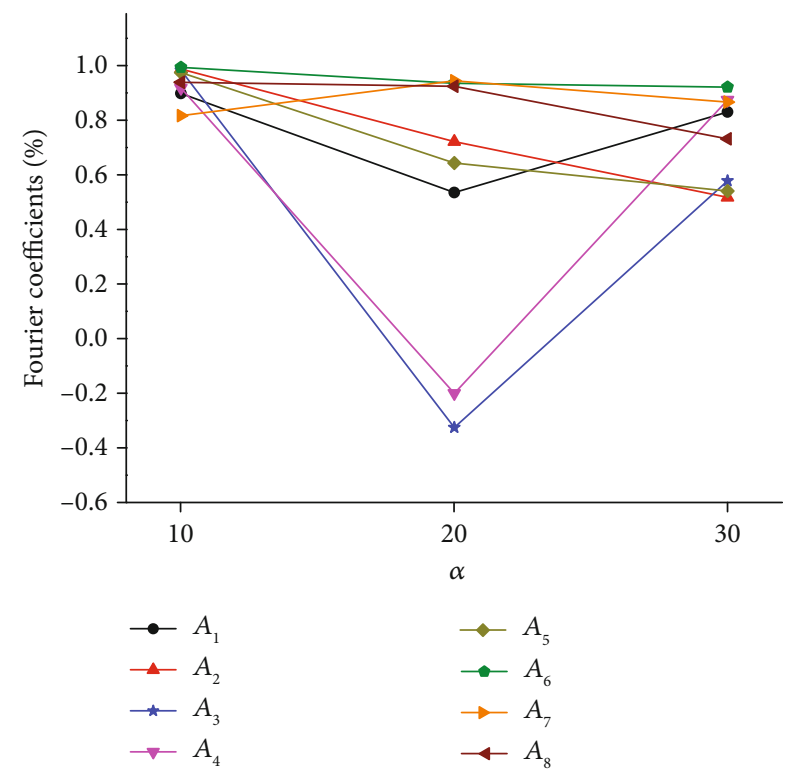

Figure 26: Percentage change of the Fourier coefficient.

than 0.99 , indicating that the fitting results of the 8 th-order Fourier series are accurate. The high-frequency variation of the lateral force coefficient of the missile body occurred by the interference of the tail fin with the missile body.

Now, subtracting Figure 24 from Figure 25, the amount of interference of the tail fin against the lateral force coefficient of the missile body could be obtained (Figure 26). When the angles of attack were $10^{\circ}$ and $30^{\circ}$, the corresponding Fourier series frequency varied similarly. However, when the angle of attack was $20^{\circ}$, the changes in the amplitudes of $A_{3}$ and $A_{4}$ were negative and the change in the amplitude of $A_{1}$ was also less than that of the other two angles of attack. Therefore, the influence of the tail fin on the lateral force of the missile body the angle of attack of $20^{\circ}$ was different from that of other angles of attack, causing a change in the direction of the Magnus moment.

\section{Conclusion}

The Magnus moment characteristics of rotating missiles with Mach numbers of 1.3 and 1.5 at different altitudes and angles of attack were numerically simulated based on the transition SST model. The main observations are presented below.

(1) The Magnus moment direction of the rotating missiles changed with the increase of the angle of attack. When the altitude was low, the Magnus moment direction changed from positive to negative with the increase of the angle of attack. At higher altitudes, the Magnus moment direction changed from positive to negative and then again to positive with the increase of the angle of attack

(2) When the angle of attack was $20^{\circ}$, the interference of the tail fin to the lateral force of the missile body was different from that for other angles of attack, leading to an increase of the lateral force of the rear part of the missile body. With the increasing altitude, the position of the boundary layer with a larger thickness of the missile body moved forward, making the lateral force distribution of the missile body even. Consequently, Magnus moments generated by different boundary layer thicknesses at the front and rear of the missile body decreased and the Magnus moment generated by the tail fin became larger. As lateral force directions of the missile body and the tail were opposite, the Magnus moment direction changed noticeably

(3) Under a high angle of attack, the Magnus moment direction changed with the increase of the angle of attack. The absolute value of the pitch moment coefficient of the missile body decreased with the increasing altitude

\section{Data Availability}

The data [[9] Jenke LM, Experimental roll-damping, Magnus, and static-stability characteristics of two slender missile configurations at high angles of attack (0 to $90 \mathrm{deg}$ ) and Mach numbers 0.2 through 2.5[R], Arnold Engineering Development Center, Tullahoma, TN, 1976] used to support the findings of this study are included within the article. The data [Microsoft Word] used to support the findings of this study are included within the supplementary information file(s) (available here).

\section{Conflicts of Interest}

The authors declare that they have no conflicts of interest. 


\section{Supplementary Materials}

These data are the variation of aerodynamic parameters of spinning missile with altitude when Mach number is 1.3 and 1.5. (Supplementary Materials)

\section{References}

[1] W. B. Sturek, H. A. Dwyer, L. D. Kayser, C. J. Nietubicz, R. P. Reklis, and K. O. Opalka, "Computations of Magnus effects for a yawed, spinning body of revolution," AIAA Journal, vol. 16, no. 7, pp. 687-692, 1978.

[2] R. Cayzac, E. Carette, P. Denis, and P. Guillen, "Magnus effect: physical origins and numerical prediction," Journal of Applied Mechanics, vol. 78, no. 5, article 051005, 2011.

[3] M. Pechier, P. Guillen, and R. Cayzac, "Magnus effect over finned projectiles," Journal of Spacecraft and Rockets, vol. 38, no. 4, pp. 542-549, 2001.

[4] J. Yin, X. Wu, J. Lei, T. Lu, and X. Liu, "Canards interference on the Magnus effect of a fin-stabilized spinning missile," Advances in Mechanical Engineering, vol. 10, no. 7, 2018.

[5] W. H. Curry and J. C. Uselton, "Some comments on the aerodynamic characteristics of the Tomahawk sounding rocket," in Sounding rocket vehicle technology specialist conference, Williamsburg, VA, 1969.

[6] Y. Zhai, G. Tao, and M. Dang, "Study on aerodynamic force and ballistic characteristics of tail stabilized rocket," Journal of Missile and Missile Guidance, vol. 31, no. 2, pp. 142-144, 2011.

[7] M. Dang, Z. Zhu, and J. Wang, "Influence of altitude on aerodynamic characteristics of missile and arrow weapons," Journal of Missile and Guidance, vol. 32, no. 4, pp. 170-172, 2012.

[8] Z. Yanwei, L. Wang, S. Chang, and J. Fu, "Stability analysis of periodic solution of nonlinear angular motion of missile and arrow," Journal of Ballistic Science, vol. 27, no. 3, pp. 7-11, 2015.

[9] Y. Zhong, L. Wang, F. Jian, and S. Chang, "Hopf bifurcation analysis of nonlinear angular motion stability of missile and arrow," Journal of Ordnungology, vol. 36, no. 7, pp. 11951202, 2015.

[10] Z. Liu, J. Han, S. Zhang, and Y. Li, "Stability analysis of the use of arc-wing rockets in plateau," Journal of Missile and Guidance, vol. 35, no. 2, pp. 79-80, 2015.

[11] P. Zhang, Z. Zhao, B. Jia, and L. Deng, "Study on the plateau attitude motion law of tail stabilized rocket," Ordnance Engineering Journal, vol. 37, no. 8, pp. 1345-1350, 2016.

[12] G. Ma, H. Zhang, H. Cai, and S. Chang, "Influence of plateau environment on dynamic stability of missile," Journal of National University of Defense Technology, vol. 42, no. 1, pp. 101-107, 2020.

[13] J. Yin, X. Wu, and J. Lei, "Body-fin interference on the Magnus effect of spinning projectile in supersonic flows," Engineering Applications of Computational Fluid Mechanics, vol. 11, no. 1, pp. 496-512, 2017.

[14] L. M. Jenke, Experimental Roll-Damping, Magnus, and StaticStability Characteristics of Two Slender Missile Configurations at High Angles of Attack (0 to $90 \mathrm{deg}$ ) and Mach Numbers 0.2 through 2.5, Arnold Engineering Development Center, Tullahoma, TN, 1976.

[15] R. Nobile, M. Vahdati, J. F. Barlow, and A. Mewburn-Crook, "Unsteady flow simulation of a vertical axis augmented wind turbine: a two- dimensional study," Journal of Wind Engineering and Industrial Aerodynamics, vol. 125, pp. 168-179, 2014.

[16] R. Langtry and F. Menter, Transition Modeling for General CFD Applications in Aeronautics, Aiaa Aerospace Sciences Meeting \& Exhibit, 2015.

[17] S. Ju, C. Yan, and Z. Ye, "Promotion and validation of transition model of $\gamma-\mathrm{Re}_{\theta t}-\mathrm{CF}$ in spalart-allmaras turbulence model," Acta Aeronautica Sinica, vol. 38, no. 4, 2017.

[18] S. Shi, J. Huo, J. Zhan, and Y. Shi, "Study on airfoil surface flow based on the model of $\gamma-\operatorname{Re}_{\theta t}$ transition," Journal of Guilin Institute of Aerospace Technology, vol. 3, pp. 334-338, 2018.

[19] S. M. Harmon, Stability Derivatives of Thin Rectangular Wings at Supersonic Speeds: Wing Diagonal Ahead of Tip Mach Lines, Technical Report Archive \& Image Library, 1948. 